\title{
Health utilities in evaluating intervention in the sleep apnoea/hypopnoea syndrome
}

\author{
I. Chakravorty*, R.M. Cayton", A. Szczepura"
}

Health utilities in evaluating intervention in the sleep apnoealhypopnoea syndrome. I. Chakravorty, R. M. Cayton, A. Szczepura. (C) ERS Journals Ltd 2002.

ABSTRACT: Formulating a rational health policy necessitates the ability to compare between different healthcare interventions and disease scenarios. Continuous positive airway pressure (CPAP) therapy with a conservative lifestyle strategy in sleep apnoea/ hypopnoea syndrome (SAHS) was evaluated using health utility and quality-adjusted life years (QALYs) as outcome measures.

A total 71 SAHS (apnoea/hypopnoea index $\geqslant 15 \mathrm{~h}^{-1}$ ) patients completed a randomised, parallel group study over 3 months using utilities derived by the standard gamble approach (Usg) and European quality of life questionnaire (Euroqol) (Ueq).

The severely impaired health status at baseline improved by $23 \%$ (Usg 0.32 to 0.55 ) adding 8 QALYs in the CPAP group, compared to a 4\% improvement with 4.7 QALYs added in the lifestyle group (Usg 0.31 to 0.35 ). The Ueq showed a marginal change with CPAP (0.73 to 0.77 ) but did not demonstrate any improvement with lifestyle intervention.

The health status impairment in sleep apnoea/hypopnoea syndrome patients is markedly improved by continuous positive airway pressure compared to a modest improvement with conservative lifestyle strategies using the standard gamble utility, which may be incorporated in effectiveness and economic analyses. The European quality of life questionnaire did not reflect a similar degree of impact and is probably not useful in this population.

Eur Respir J 2002; 20: 1233-1238.

\begin{abstract}
*Dept of Thoracic Medicine, Watford General Hospital, Watford, "Dept of Respiratory Physiology, Birmingham Heartlands Hospital, Bordesley Green East, Birmingham and "Centre for Health Services Studies, Warwick Business School, University of Warwick, Coventry, UK
\end{abstract}

Correspondence: I. Chakravorty, Dept of Thoracic Medicine, Watford General Hospital, Vicarage Road, Watford, UK. Fax: 4407069950024

E-mail: indranil@altavista.net

Keywords: European quality of life questionnaire

quality-adjusted life years utility

sleep apnoea

standard gamble

Received: August 232001

Accepted after revision: June 72002
The obstructive sleep apnoea/hypopnoea syndrome (SAHS) is characterised by repetitive sleep-induced partial or complete collapse of the pharyngeal airway. This leads to sleep fragmentation from repeated arousals required to reopen the airway and restore breathing [1]. The clinical consequences of SAHS are diverse and include excessive daytime sleepiness (EDS), deficits in neurocognitive function and an increased risk of morbidity and mortality from vascular diseases [2]. SAHS is also now recognised to cause significant deterioration in health-related quality of life (HRQL) [3].

The nasal continuous positive airways pressure (CPAP) device introduced in 1981, has been established as the treatment of choice for patients affected by this condition [4]. Research performed in SAHS patients on CPAP has shown an effective reduction in EDS, improvement in neurocognitive function and recently an improvement in HRQL [5, 6]. The evaluation of modern healthcare systems requires outcome measures and tools which are applicable across the disease spectrum and in different scenarios to allow relevant comparisons to be made between the impact of different health intervention strategies. Although measuring the efficacy of treatment in SAHS patients is possible with disease-specific parameters like the apnoea/hypopnoea index (AHI), there is no consistent relationship between patient symptoms and physiology. HRQL tools on the other hand, provide evidence of patient-generated outcome (and hence effectiveness of therapy) but differ widely both in the quality and quantity of response measured [7]. Thus, there is a role for uniform outcome measures with a potential to compare the overall impact of intervention in SAHS with other diseases.

An individual's self-valuation of the desirability of various health states at a specific moment in time, as embodied by the "utility" for that health state, is utilised in economic evaluation studies $[8,9]$. In most cases the "utility" is scaled to a value between 0 (death) to 1 (full health). Utilities may also be generated from HRQL rating scales such as the European quality of life instrument (Euroqol), but the standard gamble (SG) method is the classical approach to calculating utilities $[10,11]$. The quality adjusted life years (QALY) gained is generated by combining the utility value with predicted life expectancy thus incorporating both the quantity and quality of life in a generic measure of benefit [12].

The aim of this study was to measure the effectiveness of healthcare intervention in SAHS patients, comparing CPAP with lifestyle strategies using two 
methods of health utility measurement; the indirect Euroqol questionnaire-derived approach and the traditional and direct SG approach.

\section{Methods}

\section{Subjects}

Study population and baseline variables. All 141 patients referred to the Heartlands Sleep Clinic between June 1998 and October 1999 with a history of snoring and EDS [13] were invited to participate in the study excluding those with neuromuscular disorders, hypothyroidism and associated respiratory diseases. The study was approved by the Birmingham Heartlands and Solihull Hospital Research and Ethics Committee. Patients $(n=132)$ who gave written consent in accordance with the Committee's requirements then underwent inpatient overnight polysomnography. A total 71 patients with an AHI $\geqslant 15 \mathrm{~h}^{-1}$ were recruited. Thirty-seven patients were randomised to Group 1 (CPAP therapy), four failed to tolerate CPAP and one patient subsequently underwent surgery for a growth hormone secreting pituitary tumour. There were 34 patients randomised to Group 2 (Lifestyle), five patients were excluded due to health reasons (acute abdomen, chest infection and visual impairment), two patients preferred to go for surgery and six patients dropped out leaving 21 patients to complete the study. Patients completing the study in the CPAP arm (Group 1; $n=32$ ) had a higher body mass index (BMI), neck sizes and AHI than patients completing in the lifestyle arm (Group 2; $n=21$ ). However both the groups had similar age distribution, severity of EDS and arousal index (AI), as shown in table 1 .

Study design. All patients underwent an overnight inpatient polysomnography and those with an AHI $\geqslant 15 \mathrm{~h}^{-1}$ were recruited [13]. After a structured health status interview subjects were randomised to one of two treatment groups: Group 1 (CPAP therapy) or Group 2 (Lifestyle intervention). Patients in Group 1 were given a single night's home automatic CPAP titration study before being set-up on CPAP treatment.
Each treatment phase lasted 3 months and concluded with a repeat polysomnography and a health status interview. Patients receiving lifestyle intervention were completely unaware of the potential for being offered CPAP at the conclusion of the trial. Compliance data (usage at effective pressure) was automatically recorded and downloaded for patients on CPAP.

Diagnosis of sleep apnoealhypopnoea syndrome. Inpatient overnight polysomnography was performed in a dedicated two-bedded sleep laboratory with Compumedics Sleep System v5.0 (Compumedics Plc, Melbourne, Australia). The recording montage included two-channel electroencephalogram (EEG), submental electromyogram and electrooculogram, airflow by oronasal thermistor, chest and abdominal wall movement by inductive plethysmography, standard limb lead II electrocardiogram, limb movements by piezo-electric crystals and pulse oximetry (Minolta Pulsox-7; Minolta Co Ltd, Osaka, Japan). Standard criteria were used for scoring sleep [14], apnoeas/ hypopnoeas [15] and EEG arousals.

Health status interview (baseline). The diagnosis of SAHS was discussed with patients and their partners and both verbal and written information on clinical and management options provided. Subjects then completed the Euroqol and the Epworth Sleepiness Scale (ESS) questionnaires unsupervised $[10,16]$. The Euroqol measures graded responses (1=no problem, $2=$ some problem, $3=$ extreme problem) in five dimensions of health (mobility, self-care, pain, usual activities and anxiety/depression). Utility values for health states are generated by combining normative data on 243 possible combinations of responses to the five questions with preferences/weights for various health states provided by a UK population survey performed in 1993 and 1995/1996, (worst possible health state=-0.59 to full health=1) [17]. The Euroqol also incorporates a linear visual analogue scale (i.e. health thermometer) to value present health state $(0=$ worst imaginable health to $100=$ best imaginable health).

The interview included a simple explanation of the SG method [11] and patients were asked to choose either to remain in their current state of health "I" or

Table 1. - Characteristics of the patients in Groups 1 and 2 at baseline

\begin{tabular}{|c|c|c|c|c|}
\hline & \multicolumn{2}{|c|}{ Group 1} & \multicolumn{2}{|c|}{ Group 2} \\
\hline & CPAP & Post-treatment & Lifestyle & Post-treatment \\
\hline Subjects $n$ & 32 & & 21 & \\
\hline Age yrs & $49 \pm 11$ & & $52 \pm 9.6$ & \\
\hline $\mathrm{BMI} \mathrm{kg} \cdot \mathrm{m}^{-2}$ & $40 \pm 14.5$ & $40 \pm 12.8$ & $32.3 \pm 5.5$ & $31.7 \pm 5.6^{*}$ \\
\hline Neck size cm & $50 \pm 6.4$ & $48 \pm 5.8$ & $45 \pm 5.6$ & $45 \pm 5.8$ \\
\hline $\mathrm{AHI} \cdot \mathrm{h}^{-1}$ & $55 \pm 28.7$ & $8 \pm 28 * * *$ & $35 \pm 19.1$ & $34 \pm 21$ \\
\hline ESS (0-24) & $16 \pm 5.6$ & $8 \pm 6.4 * * *$ & $14 \pm 4.2$ & $11 \pm 5^{*}$ \\
\hline $\mathrm{U}_{\mathrm{sg}}$ & $0.32 \pm 0.17$ & $0.55 \pm 0.26^{*}$ & $0.31 \pm 0.13$ & $0.35 \pm 0.12 *$ \\
\hline Ueq & $0.73 \pm 0.18$ & $0.77 \pm 0.18^{*}$ & $0.77 \pm 0.12$ & $0.77 \pm 0.09$ \\
\hline Euroqol thermometer $(0-100)$ & $59 \pm 19.8$ & $70 \pm 18.1^{*}$ & $68 \pm 16.8$ & $68 \pm 18.6$ \\
\hline
\end{tabular}

Data are presented as mean \pm SD. BMI: body mass index; AHI: apnoea/hypopnoea index; Usg: standard gamble utiltiy; Ueq: European quality of life questionnaire (Euroqol) derived utility. $*: \mathrm{p}<0.05 ; * * * \mathrm{p}<0.001$. 
to be treated. The treatment had two outcomes; either complete cure resulting in best possible health $(\mathrm{I}=1)$ or failure leading to a much worse state of health/death $(I=0)$. The probability of success $(p)$ or failure $(1-p)$ was varied using a probability wheel, until a point of indecision was reached. The probability $\left(p^{*}\right)$ of success at this "point of indecision" was used to calculate the value/utility of health between 0 and 1 ( $\left.\mathrm{Usg}_{\mathrm{sg}}=\mathrm{p}^{*}\right)$.

Group 1: continuous positive airway pressure therapy. Patients who were randomised to receive CPAP therapy underwent a home-based CPAP titration study using the Autoset II CPAP system (ResMed Plc, Abingdon, Oxford, UK). This system used a validated algorithm based on the flattening of the inspiratory flow curve to detect obstructive apnoeas and hypopnoeas and automatically titrate the CPAP pressure to eradicate these events [18]. A 90th percentile pressure predicted by the Autoset II titration study, was then used for initial setting of CPAP machines (Sullivan V Elite, ResMed Plc). Fitting of appropriate masks and demonstration of the use of CPAP machines was provided by an experienced technician followed by a 30 -min trial in the laboratory. Subjects were given direct access to the technicians and encouraged to ring in case of any difficulties.

Group 2: lifestyle intervention. Each patient (along with their partners) received standard verbal advice and an explanation of the intention to measure the effect of a conservative strategy on snoring, daytime symptoms and nocturnal sleep disruption. A written leaflet listing the strategies for sleep hygiene, quitting smoking, reducing alcohol consumption and controlling stress was provided and discussed. After recording body weights and neck measurements they were interviewed by a hospital dietitian. Verbal and written advice was provided on ideal body weight, weight reducing diet and regular exercise. Follow-up appointments were arranged at 4 and 8 weeks. Patients were asked to maintain a sleep diary with total number of hours slept, and a visual analogue scale self-assessment of their daily quality of sleep and daytime alertness score.

\section{Statistical analysis}

The primary outcome measure was the number of QALYs gained by treatment with CPAP compared to lifestyle intervention. The change in health utility $(\delta)$ measured by the SG approach and the Euroqol and the health thermometer value were compared between the groups, along with the change in ESS scores. The relationship between the HRQL and physiological variables was also assessed. Data is expressed as mean and SD. An independent t-test was used at baseline between Group 1 and Group 2. The treatment effect was analysed using the paired samples t-test. The correlation between utility values and disease/demographic parameters were measured using Spearman's rho. The corrected mean $\delta$ utility values were evaluated using a multiple linear regression model to adjust for the age, sex, BMI, AHI and neck size between the groups. This was then multiplied by the life expectancy of each patient based on the UK 1996 population census [19] to generate the QALYs gained by intervention in each treatment group.

\section{Results}

Patients recruited to the study had a mean \pm SD: age $50 \pm 11 \mathrm{yrs}$; BMI $37 \pm 12 \mathrm{~kg} \cdot \mathrm{m}^{-2}$; evidence of moderate daytime sleepiness, ESS score $14 \pm 5$; respiratory and sleep disturbance, AHI $49 \pm 28 \mathrm{~h}^{-1}$; and AI $61 \pm 30 \mathrm{~h}^{-1}$. Baseline health status assessment using the $\mathrm{SG}$ approach showed a very low valuation of general health in both groups of patients (Group 1: $\mathrm{Usg}_{\mathrm{sg}}=0.32$; Group 2: $\left.\mathrm{Usg}_{\mathrm{sg}}=0.31\right)$. However the Euroqol derived health utility (Group 1: Ueq=0.73; Group 2: Ueq=0.77) was higher than Usg in both groups of patients, shown in table 1 .

Three months after intervention, patients on CPAP reported a $23 \%$ improvement in general health status (Usg 0.32 to $0.55, \mathrm{p}=<0.001$ ) compared to $4 \%$ in Group 2 (Usg 0.31 to $0.35, \mathrm{p}<0.001$ ). The Euroqol derived utility showed a modest 4\% improvement $(\mathrm{p}<0.001)$ in the health status among subjects in Group 1 but no change in Group 2. The Euroqol health thermometer scores showed an $11 \%$ improvement after CPAP use in Group $1(\mathrm{p}<0.001)$ but no improvement in Group 2.

Subjects in Group 2 achieved a mean reduction of body weight by $1.85 \mathrm{~kg}$ (range $-3.8-5.9 \mathrm{~kg}$ ) while their mean BMI dropped to 31.7 from $32.3 \mathrm{~kg} \cdot \mathrm{m}^{-2}$ $(p<0.05)$. There was no change in the BMI or body weight of patients in the CPAP group.

Among Group 1 patients the sleep latency increased, total sleep time decreased and there were significant reductions in the arousal index and mean oxygen desaturation (table 2) as well as AHI and ESS (table 1). However, Group 2 patients demonstrated a reduction in AHI during rapid eye movement (REM) sleep (which reduced from $42 \pm 17.7$ to $26 \pm 19.6 \mathrm{~h}^{-1}$, $\mathrm{p}<0.05)$, arousal index and ESS scores $(\mathrm{p}<0.05)$.

It was estimated that 8.2 QALYs were gained by intervention in patients treated with CPAP compared to 4.7 QALYs in Group 2, using the SG approach $(\mathrm{p}<0.001)$. There were 1.4 QALYs gained in Group 1, based on Euroqol-derived utility compared to no significant change demonstrated in Group $2(\mathrm{p}<0.001)$, as shown in table 3.

Analysis of the self-reported sleep diary data amongst patients in Group 2 showed no change in mean total hours of sleep in a 24-h period and daytime sleepiness using a visual analogue scale, post-treatment but only a $5 \%$ improvement in visual analogue scale for sleep quality, $\mathrm{p}<0.05$.

Baseline health status was not correlated with any of the variables considered and 3 months on lifestyle intervention did not significantly alter the health status. However in the CPAP group, the change in utility $(\delta s g)$ correlated with baseline body habitus, measures of respiratory disturbance during sleep (AHI and arterial oxygen desaturation index) and the change in ESS score on treatment (table 4).

The change in Euroqol health thermometer scores 
Table 2. - Polysomnographic variables at baseline and post-treatment

\begin{tabular}{|c|c|c|c|c|}
\hline \multirow[t]{2}{*}{ Variables } & \multicolumn{2}{|c|}{ Group 1} & \multicolumn{2}{|c|}{ Group 2} \\
\hline & Baseline & Post & Baseline & Post \\
\hline Sleep latency min & $19.0 \pm 23.7$ & $33.9 \pm 38.8^{*}$ & $24.8 \pm 29.4$ & $40 \pm 38.2$ \\
\hline Stage $1+2 \min$ & $180(62.5)$ & $166.2(69.4)$ & $221.9(73.3)$ & $211.6(90)$ \\
\hline Stage $3+4 \mathrm{~min}$ & $64.7(50.2)$ & $69.5(50.1)$ & $81.2(52.5)$ & $85.3(46.6)$ \\
\hline REM latency min & $125.8(81.5)$ & $105.7(72)$ & $155.7(85.7)$ & $114.6(43.1)$ \\
\hline REM period min & $80.6(39.3)$ & $81.9(35.8)$ & $87.1(40.5)$ & $92.5(47.8)$ \\
\hline Total sleep time min & $389.9(113.4)$ & $329.2(86)^{*}$ & 434 (116.8) & $387(83.1)$ \\
\hline Sleep efficiency $\%$ & $67.7(18.2)$ & $67.5(15.7)$ & $71.6(12.8)$ & $74.4(11.1)$ \\
\hline Arousal index $\cdot h^{-1}$ & $64(28)$ & $38.9 \pm 28.6^{* * *}$ & $52.2(28.5)$ & $36.9(21.5)^{*}$ \\
\hline Mean $S_{\mathrm{a}, \mathrm{O}_{2}}$ desaturation $\%$ & $6.9(5.4)$ & $3(2.1)^{*}$ & $5.7(5.8)$ & $6.9(5.3)$ \\
\hline
\end{tabular}

Data are presented as mean \pm SD. REM: rapid eye movement; $\mathrm{Sa}_{\mathrm{a}} \mathrm{O}_{2}$ : arterial oxygen saturation; Sleep efficiency: sleep period/ time in bed; Group 1: continuous positive airways pressure; Group 2: lifestyle. *: $\mathrm{p}<0.05$; ***: $\mathrm{p}<0.001$.

Table 3. - Change in utility $(\delta)$ and quality-adjusted life years (QALYs) gained with treatment

\begin{tabular}{|c|c|c|c|c|}
\hline \multirow[t]{2}{*}{ Utility } & \multicolumn{2}{|c|}{ Mean $\delta$} & \multicolumn{2}{|c|}{ Mean QALY } \\
\hline & Uncorrected & Corrected & Uncorrected & Corrected \\
\hline \multicolumn{5}{|l|}{$U_{s g}$} \\
\hline Group 1 & $0.24 \pm 0.23$ & $0.23 \pm 0.09$ & $7.9 \pm 7.8$ & $8.2 \pm 5.2$ \\
\hline Group 2 & $0.04(0.12)$ & $0.15(0.05)$ & $0.8(3.8)$ & $4.7(2.2)^{* * *}$ \\
\hline \multicolumn{5}{|l|}{ Ueq } \\
\hline Group1 & $0.04(0.1)$ & $0.04(0.04)$ & $1.5(3.3)$ & $1.4(1.7)$ \\
\hline Group 2 & $0.006(0.1)$ & $0.007(0.02)$ & $0.03(3.0)$ & $0.27(0.53)^{* * *}$ \\
\hline
\end{tabular}

Data are presented as mean \pm SD. Usg: standard gamble utility; Ueq: European quality of life questionnaire-derived utility; Group 1: continuous positive airways pressure; Group 2: lifestyle. The raw utilities were corrected using a multiple linear regression model to adjust baseline differences in body mass index, apnoea/hypopnoea index and arterial oxygen desaturation index to yield values predicted by the equation including all the above variables. These predicted values were used in calculating the "corrected mean" and hence were adjusted for baseline differences. Significance tests were used for intergroup differences. ${ }^{* * *}: \mathrm{p}<0.001$.

showed a negative correlation with baseline scores (Spearman's rho=-0.5, p=0.009) but demonstrated a positive correlation to change in ESS scores (Spearman's rho $=0.49, \mathrm{p}=0.02$ ), indicating that the lower baseline health perception, the larger change on treatment.

Table 4. - Significant correlations (Spearman's rho) for the difference in utility by the standard gamble approach in Group 1

\begin{tabular}{lcc}
\hline Variable & Correlation coefficient & p-value \\
\hline ODI h$^{-1}$ & 0.66 & $<0.001$ \\
Difference in ESS scores & 0.59 & $<0.05$ \\
BMI baseline $\mathrm{kg} \cdot \mathrm{m}^{-2}$ & 0.58 & $<0.05$ \\
Baseline neck size cm & 0.54 & $<0.05$ \\
Baseline weight kg & 0.53 & $<0.05$ \\
Baseline AHI $\cdot \mathrm{h}^{-1}$ & 0.45 & $<0.05$ \\
CPAP usage $\cdot$ night $^{-1}$ & 0.49 & $<0.05$ \\
\hline
\end{tabular}

ODI: arterial oxygen desaturation index; ESS: Epworth Sleepiness Scale; BMI: body mass index; AHI: apnoea/ hypopnoea index; CPAP: continuous positive airways pressure. Age, life expectancy, baseline utility, baseline ESS score and electroencephalogram arousal index were not significant.

\section{Discussion}

When asked to assess their health status using a direct approach incorporating the concept of risk with the SG method, SAHS patients demonstrated a low baseline value and a large improvement with CPAP treatment compared to conservative lifestyle measures. This overall change in the "quality" of health status when combined with the life expectancy (quantity) in the QALYs yields a measure of effectiveness which is generic and thus allows comparison between different health intervention measures and disease scenarios facilitating health resource allocation [20]. Using an alternative indirect utility measurement with the five item-based Euroqol questionnaire, assessing on a scale from 1-3 aspects such as mobility, self-care, pain, usual activities and anxiety/depression, the baseline health status was higher, the improvement with CPAP in these parameters only minor and there was no change demonstrated with conservative strategies.

This study focused on comparing a widely implemented traditional direct approach in measuring health status (SG) with a questionnaire-derived indirect utility assessment tool (Euroqol) that is widely used in Europe and around the world. It was found 
that the baseline utilities in untreated SAHS were comparable with those seen in patients with a major stroke (0.32-0.41) and much worse than prostatic cancer with sexual and urinary dysfunction and minor stroke $(0.72)$ [21, 22].

In a Canadian study, Tousignant et al. [23] reported 5.4 QALYs added by CPAP treatment in a series of 19 patients in a retrospective analysis using the SG approach, where the health status improved from 0.63 to 0.87 , a net change of 0.24 . Interestingly the patients in the present study reported a similar degree of improvement, but from a more severely impaired state to a more modest health state. As all of the Canadian patients had already been established on CPAP treatment for a considerable period of time, and were then asked to retrospectively value their pretreatment health status, substantial differences might be anticipated with the present prospective, randomised and controlled approach. The primary methodological weakness in the Canadian retrospective study arises from the most likely failure of "well-treated" patients to remember their previously impaired state accurately, hence perhaps the higher baseline health state, and the tendency for selfreporting to overestimate any change on treatment, in the absence of a control group.

Firstly, the present results confirm the findings that correcting for interactions with traditional confounding variables like age, sex, BMI and AHI CPAP therapy, has a clear effectiveness among SAHS patients in improving their net valuation of general health status. Secondly, these results suggest that the Euroqol questionnaire, which is widely used in generating QALYs, effectiveness assessments and in implementation of health policy may not be an ideal instrument for use among SAHS patients. Even among moderate to severely disabled SAHS patients, Euroqol as a health status tool showed only a mild change in patients with an effective positive treatment response to CPAP and failed to record the mild improvement in the lifestyle intervention group. No association between disease severity and health status at baseline and after treatment in both the groups was found. In a previous analysis of Euroqol as an outcome measure in SAHS, JENKINSON et al. [7] reported a higher pretreatment health status compared to the Short-Form 36 questionnaire and a lack of sensitivity in measuring the impact of treatment. This failure to discriminate between treatment effects among patients on CPAP and lifestyle strategy may result from the indirect derivation of utilities based on a scaled response to mobility, self-care, usual activity, pain/discomfort and anxiety/depression. This fivedimensional model most certainly excludes dimensions which are likely to be sensitive to the health impairment in SAHS such as energy/vitality and social functioning affected by tiredness and daytime sleepiness. The more direct visual analogue scale incorporated in the Euroqol questionnaire (health thermometer) showed an $11 \%$ improvement in health state after treatment, in the CPAP group. Thus, the lack of sensitivity of Euroqol in this population may have an impact on health resource planning using such instruments.
The goal of treatment in SAHS patients has traditionally focused on improvements in daytime symptoms and function. Although severity of SAHS is defined by the evidence of nocturnal respiratory disturbance in the presence of significant symptoms, there is only a very weak relationship between the AHI $(r=0.4)$ [24] and response to treatment. Hence measures such as the AHI and AI do not lend themselves for use as good outcome measures to assess the effectiveness of therapy. In a study with moderate-to-severe SAHS patients, BALLESTER et al. [6] compared 37 patients on a 3-month conservative therapy (sleep hygiene and weight loss) programme with 68 patients on conservative plus CPAP therapy. Their results demonstrated the odds for experiencing a treatment response in the CPAP plus conservative therapy group (combining ESS plus three dimensions of the Short-Form 36 questionnaire) as $6.5(95 \%$ confidence interval 2.5-17.6). HRQL has a multidimensional approach and hence the response to therapy recorded with individual tools varies between the various dimensions like energy/vitality from ShortForm 36 to mental health. There is also a lack of a consistent relationship between the HRQL measures and nocturnal physiological events. A consistent and reproducible linear relationship between the improvement in the dimensions of HRQL affected and the physiological parameters of disease severity is not yet identified. Hence the role for a generic instrument, such as the SG, which measures the net effect of treatment on overall health status is suggested, as this is applicable to different patient groups and healthcare scenarios.

A modest contribution of lifestyle intervention measures in health status improvement among SAHS patients was found, although this is considered to be the first-line strategy by many clinicians. There was a clear trend of improvement in polysomnographic parameters especially in daytime somnolence, AHI during REM sleep and AI after introduction of lifestyle and sleep hygiene measures, though other parameters i.e. mean AHI, sleep latency, sleep efficiency did not reach statistical significance. The high dropout rate among this group suggested the inherent difficulty in achieving a real change in lifestyle among tired and disenchanted patients and probably a mismatch between the patient expectation and benefits derived. Patients on lifestyle measures were deliberately not informed of any alternative future measures like the CPAP, which may be offered if these measures failed in order to ensure a commitment to lifestyle changes, hence may have contributed to their failure to complete the trial. The reduced number of patients completing the trial in Group 2 is likely to have reduced the power of this study to significantly measure such changes. Others have reported a weight loss of $1.1-3.1 \mathrm{~kg}$ among their patients with a similar 3-month programme $[6,24]$. Poor results have been previously reported for conservative weight loss strategies, while the only substantial improvement in disease severity was achieved by gastro-jejunal surgery [25]. It has been postulated that in SAHS sufferers a lack of motivation, energy and vitality can make it difficult to achieve clinically significant loss in body 
weight. This was certainly the case in the authors' experience.

\section{Conclusion}

To conclude, sleep apnoea/hypopnoea syndrome sufferers experience severe health impairment and therefore, have a low valuation of their health status, which improves remarkably with continuous positive airway pressure therapy compared to marginal improvement with a lifestyle intervention strategy, (including weight-loss programmes). The standard gamble approach to measuring health utility clearly demonstrates the impact of healthcare intervention strategies, while the European quality of life questionnaire showed only a much lower magnitude of change with continuous positive airway pressure and did not measure the change with lifestyle strategy. Hence, the European quality of life questionnaire is perhaps an inappropriate tool for use in assessing outcome in a sleep apnoea/hypopnoea syndrome population. Utility values derived from the standard gamble approach should allow comparison of continuous positive airway pressure intervention with other interventions in healthcare.

\section{References}

1. White DP. Sleep-related breathing disorder. 2. Pathophysiology of obstructive sleep apnoea. Thorax 1995; 50: 797-804.

2. Ferguson KA, Fleetham JA. Sleep-related breathing disorders. 4. Consequences of sleep disordered breathing. Thorax 1995; 50: 998-1004.

3. D'Ambrosio C, Bowman T, Mohsenin V. Quality of life in patients with obstructive sleep apnoea: effect of nasal continuous positive airway pressure - a prospective study. Chest 1999; 115: 123-129.

4. Sullivan CE, Issa FG, Berthon-Jones M, Eves L. Reversal of obstructive sleep apnoea syndrome by continuous positive airway pressure applied through the nares. Lancet 1981; 1: 862-865.

5. Engleman HM, Kingshott RN, Wraith PK, MacKay TW, Deary IJ, Douglas NJ. Randomised placebo-controlled crossover trial of continuous positive airway pressure for mild Sleep apnoea/ hypopnoea syndrome. Am J Respir Crit Care Med 1999; 159: 461-467.

6. Ballester E, Badia JR, Hernandez L, et al. Evidence of the effectiveness of continuous positive airway pressure in the treatment of sleep apnea/hypopnea syndrome. Am J Respir Crit Care Med 1999; 159: 495-501.

7. Jenkinson C, Stradling J, Petersen S. Comparison of three measures of quality of life outcome in the evaluation of continuous positive airways pressure therapy for sleep apnoea. J Sleep Res 1997; 6: 199-204.

8. Torrance GW, Thomas WH, Sackett DL. A utility maximization model for evaluation of health care programs. Health Serv Res 1972; 7: 118-133.
9. Sackett DL, Torrance GW. The utility of different health states as perceived by the general public. J Chronic Dis 1978; 31: 697-704.

10. Euroqol Group. A new facility for the measurement of health related quality of life. Health Policy 1990; 16 : 199-208.

11. Gafni A. The standard gamble method: what is being measured and how it is interpreted. Health Serv Res 1994; 29: 207-224.

12. Weinstein MC, Stason WB. Foundations of costeffectiveness analysis for health and medical practices. N Engl J Med 1977; 296: 716-721.

13. Gould GA, Whyte KF, Rhind GB, et al. The sleep hypopnoea syndrome. Am Rev Respir Dis 1988; 137: 895-898.

14. Rechtschaffen A, Kales A. A manual of standardized terminology, techniques and scoring system for sleep stages of human subjects. Washington DC, US Government Printing Office, 1968.

15. American Academy of Sleep Medicine. Sleep-related breathing disorders in adults: Recommendations for syndrome definition and measurement techniques in clinical research. Sleep 1999; 22: 667-689.

16. Johns MW. A new method for measuring daytime sleepiness: the Epworth Sleepiness Scale. Sleep 1991; 14: $540-545$.

17. Dolan P, Gudex C. Time preference, duration and health state valuations. Health Econ 1995; 4: 289-299.

18. Teschler H, Berthon-Jones M, Thompson AB, Henkel A, Henry J, Konietzko N. Automated continuous positive airway pressure titration for obstructive sleep apnea syndrome. Am J Respir Crit Care Med 1996; 154: 734-740.

19. Department of Health \& Human Resources. Life expectancy tables. London, UK Government Publication. 1996.

20. Patrick DL, Erickson J. Health status and health policy; Quality of life in health care evaluation and resource allocation. New York, Oxford University Press, 1993.

21. Post PN, Stiggelbout AM, Wakker PP. The utility of health states after stroke: a systematic review of the literature. Stroke 2001; 32: 1425-1429.

22. Smith DS, Krygiel J, Nease RF Jr, Sumner W, Catalona WJ. Patient preferences for outcomes associated with surgical management of prostate cancer. J Urol 2002; 167: 2117-2122.

23. Tousignant P, Cosio MG, Levy RD, Groome PA. Quality adjusted life years added by treatment of obstructive sleep apnea. Sleep 1994; 17: 52-60.

24. Bennett LS, Barbour C, Langford B, Stradling JR, Davies RJ. Health status in obstructive sleep apnea: relationship with sleep fragmentation and daytime sleepiness, and effects of continuous positive airway pressure treatment. Am J Respir Crit Care Med 1999; 159: $1884-1890$

25. Noseda A, Kempenaers C, Kerkhofs M, Houben JJ, Linkowski P. Sleep apnea after 1 year domiciliary nasal-continuous positive airway pressure and attempted weight reduction. Potential for weaning from continuous positive airway pressure. Chest 1996; 109: 138-143. 